\title{
Current biomarker-associated procedures of cancer modeling-a reference in the context of IDH1 mutant glioma
}

\author{
Narges Zare Mehrjardi ${ }^{1}$, Daniel Hänggi ${ }^{1}$ and Ulf Dietrich Kahlert ${ }^{1}$
}

\begin{abstract}
Isocitrate dehydrogenases (IDH1/2) are central molecular markers for glioblastoma. Providing in vitro or in vivo models with mutated IDH1/2 can help prepare facilities to understand the biology of these mutated genes as glioma markers, as well as help, improve therapeutic strategies. In this review, we first summarize the biology principles of IDH and its mutations and outline the core primary findings in the clinical context of neuro-oncology. Given the extensive research interest and exciting developments in current stem cell biology and genome editing, the central part of the manuscript is dedicated to introducing various routes of disease modeling strategies of IDH mutation (IDH ${ }^{\text {Mut }}$ glioma and comparing the scientific-technological findings from the field using different engineering methods. Lastly, by giving our perspective on the benefits and limitations of patient-derived and donor-derived disease modeling respectively, we aim to propose leading research questions to be answered in the context of IDH1 and glioma.
\end{abstract}

\section{Facts}

- Mutations in IDH genes represent the first central genomic markers to guide clinical diagnosis in neuro-oncology.

- The involvement of IDH mutations in various processes of glioma cell biology opens an opportunity for new treatment and diagnostic strategies, with several clinical trials underway.

- Classical in vitro disease modeling in the context of IDH mutant brain tumors using patient-derived systems is challenging given the general disadvantages in terms of cell growth and survival.

- Novel strategy of in vitro cancer modeling using the transformation of healthy donor-derived stem cells is emerging to combat this hurdle.

\section{Open questions}

- Can single cell-derived, synthetic cancer in vitro modeling based on the transformation of healthy donor-derived cells with disease resembling genetic elements overcome hurdles experienced when relying on cancer cell lines only (i.e. limitations in reproducibly of research results, a limited accurate recapitulation of pathophysiology)?

- Since IDH1 mutations occur in other cancers than brain tumor, can the progress of understanding the glioma IDH1 mutation cell biology translate into tumor-agnostic approaches?

- Can the interrogation of technological advanced cell model systems in in vitro screening approaches justify the prolonged establishment of such explorative experimental design in the early stage of current drug development processes?

\section{Introduction}

Mutations in the DNA encoding for isocitrate dehydrogenases (IDH) mutations are recognized as one of the 
main molecular markers in $70-80 \%$ of stage II or III astrocytomas, oligodendrogliomas, and secondary GBMs, as opposed to primary tumors ${ }^{1}$. In the WHO classification, expressions of mutated IDH1/2, TP53, chromatin remodeling, and a loss of alpha-thalassemia/mental retardation, X-linked (ATRX) has been seen in astrocytoma. In contrast, oligodendrogliomas carry mutated IDH1/2 cells associated with $1 \mathrm{p} / 19 \mathrm{q}$ co-deletion and TERT promoter mutations ${ }^{2,3}$. The biology of IDHs in cancer is not fully understood, but several therapeutic opportunities arise as a result of this molecular alteration. To better understand and develop precision medicine approaches to IDH mutation (IDH ${ }^{\text {Mut }}$ ) relevant modeling tools are needed. Despite the technical advances in modern biomedicine, the field is suffering from a lack of pathophysiological relevant modeling systems to study the disease in a controlled experimental setting. In this review, we focus on current disease modeling efforts for one of the most studied genes linked to deadly cancer. To our knowledge, this is the hitherto most comprehensive reference describing technical details of successful cell engineering protocols that assess their practicality and applicably from an academic lab perspective. By including a listing of the main biological findings made with different lab tools and comparing them to the patient scenario, we also propose a ranking of their pathophysiological relevance. Given the comprehensive nature of our review that examines the details of current stem cell technologies, molecular editing, and in vitro pharmacometabologenomics, we anticipate that the significance of this review will extend well beyond the field of neurooncology.

\section{Principles of IDH function and pathological findings in a clinical context of IDH1 mutation (IDH1 ${ }^{\text {Mut }}$ ) in neuro-oncology}

IDH is a small protein that expresses primarily in the liver, heart muscle, and skeletal muscle ${ }^{4}$. There are five genes that encode IDHs: IDH1, IDH2, IDH3A, IDH3B, and IDH3G. IDH1 and IDH2 are active as homodimers, while IDH3 is active as a heterotetramer containing $2 \alpha$, $1 \beta$, and $1 \gamma$ subunits, all of which function as the electron acceptor $^{5}$. The role of mutated IDH1/2 has been reported in low-grade gliomas (LGG) ${ }^{6}$. According to the authors' knowledge, no study shows the IDH3 mutation related to any kind of cancer. For the first time, in 2006, Sjoblom et al. $^{7}$ reported the IDH1 mutation (IDH1R132C) in breast and colorectal cancers. Two years later, Parsons et al. $^{8}$ announced an IDH1-R132H mutation in gliomas. Mutated IDH1, as a common mutation, was then reported in glioma, acute myeloid leukemia (AML), cholangiocarcinoma, melanoma, and cartilaginous tumors ${ }^{8}$.

IDH mutations in gliomas are normally heterozygous missense mutations ${ }^{9}$. In addition to the $\mathrm{R} 132 \mathrm{H}$ mutation, other IDH1 mutations in glioma are R132L, R132C, R132G, R132S ${ }^{10}, \mathrm{R}_{132 \mathrm{~V}^{5} \text {, and IDH2-R172K or R140Q }} 5,11$ which all of these mutations show identical output. However, a mutation in IDH1 or IDH2 is considered an early event in gliomagenesis ${ }^{12}$; they are not classic oncogenes. It seems that these genes can facilitate prooncogene mutations, such as TP53 in IDH1-R132H astrocytoma $^{13}$, or downregulation of the expression of the genes related to immune response, which was observed in IDH1-R132H gliomas ${ }^{14}$. The protein of IDH1 localizes in the cytoplasm and peroxisome, while IDH2 localizes in the mitochondria. These two genes are (NADP1)-dependent and promote the oxidative decarboxylation of isocitrate to alpha-ketoglutarate $(\alpha-K G)$ that protects the cells from reactive oxygen species (ROS), which can cause DNA damage ${ }^{15}$. In addition, $\alpha-K G$ has been shown to serve as a cofactor for several important cellular reactions, including histone modifications, hypoxia sensing, and fatty acid metabolism ${ }^{16}$. Any mutation in IDH1 or IDH2 forces the cells to convert a-KG into the D isomer of 2-hydroxyglutarate $(\mathrm{D} 2 \mathrm{HG})^{17-20}$. Due to the effect of the D2HG in cancer, this metabolite has been called oncometabolite which can cause several epigenetic abnormalities, such as DNA demethylases, histone modification, non-coding RNA (ncRNA), microRNA (miRNA), and chromatin remodeling ${ }^{21}$. Among IDH1 mutations, $\mathrm{R} 132 \mathrm{H}$ is the most common mutation $(90 \%)$ in glioma ${ }^{22}$. In this mutation, adenine is replaced with guanine at nucleotide 395 (c.395G>A), which converts to histidine instead of arginine in protein sequencing (p.Arg132His). Pusch et al. ${ }^{23}$ clarified that this event is due to the fact that human glioma cells with R132G, $\mathrm{R} 132 \mathrm{C}$, and R132S produce higher concentrations of D2HG as compared to those with $\mathrm{R} 132 \mathrm{H}$. A high concentration of D2HG is toxic for the glioma cells and induces biological alternations such as inhibiting the proliferation and migration of these cells. Therefore, cells prefer $\mathrm{R} 132 \mathrm{H}$ mutation with a moderate amount of $\mathrm{D}_{2} \mathrm{HG}^{23}$. The concentration of D2HG in glioma cells carrying IDH1-R132H is usually between 10 and $30 \mathrm{mM}^{24}$. In addition to glioma cells, D2HG can be found in the non-neoplastic cells around the tumors in the patients carrying IDH1 mutation. Linninger et al. ${ }^{24}$ detected around $100 \mathrm{mM}$ of D2HG in the circulating cerebrospinal fluid (CSF) of LGG patient. This amount of D2HG can cause oxidative stress, inhibition of expression of pro-apoptotic proteins, reduction of pro-inflammatory signaling, and changing cellular metabolism in these patients ${ }^{24}$. Jin et al. ${ }^{18}$ have shown that the level of the D2HG cells carrying IDH1 mutation depends on the wildtype (wt) allele, however; this level in IDH2 mutated cells is related to the site of mutation. Ward et al. ${ }^{25}$ revealed that glioma cells carrying IDH2-R140K produce less D2HG in comparison to IDH2-R172K mutation. 
Although the role of heterozygous IDH1 mutation (IDH1wt-R132H) is known in glioma biology, the association of the level of D2HG and localization of it in the subcellular compartment on the abnormality of the brain cells in glioma patients is little understood ${ }^{26}$.

Recently, the cancer genome atlas (TCGA) analysis by Unruh et al. showed that IDH1wt/R132H has a special effect on the DNA-methylome and transcriptome of gliomas. A study of DNA-methylation of the patient samples with glioma demonstrated that from 365,092 analyzed CpG sites, 70,591 (19\%) were hypermethylated in gliomas carrying IDH1wt/R132H, compared to wildtype gliomas. The ratio of hypermethylation changes during differentiation leads to suppression of tissue development ${ }^{12}$ and differentiation ${ }^{27}$ of glioma cells. In addition, this mutation can hypermethylate and modify important glioma-related genes, such as EGFR and PDGFRA $^{28,29}$. Hypermethylation following the IDH1 ${ }^{\text {Mut }}$ may also lead to chromatin disorganization ${ }^{30,31}$ because mutated cells gain less condensed chromatin, leading to an increase in DNA damage ${ }^{32}$. On the other hand, IDH1 ${ }^{\text {Mut }}$ decreases self-renewal and proliferation rate in vitro and in vivo in glioma cells ${ }^{31,33}$. This can be explained by hypermethylation and downregulation of PROM1 that encodes for CD133, the bona fide stem cell marker of glioma. CD133 is the primary stem cell factor in glioma, and a determiner of growth for glioblastoma cells in immunocompromised mice, a fact that was reported to be somewhat glioma specific in the context of IDH1 mutated cancers ${ }^{12}$. Phosphoinositide 3-kinase (PI3K)/ AKT signaling is an important pathway for cell cycling and cell survival ${ }^{34}$. IDH1 ${ }^{\text {Mut }}$ inhibits the PIK3/AKT signaling in human glioma cells ${ }^{35}$. This helps further explain the declining proliferation rate of glioma cells with IDH $1^{\mathrm{wt} / \mathrm{R} 132 \mathrm{H}}$ compared to IDH ${ }^{\mathrm{wt} / \mathrm{wt}}$ cells $^{36}$. Furthermore, by overexpression of $\mathrm{IDH} 1^{\mathrm{wt} / \mathrm{R} 132 \mathrm{H}}$ in the NSC derived from human-induced pluripotent stem cells (hiPSCs), Modrek et al. showed downregulation of expression of Sox2. Sox 2 is largely responsible for NSC self-renewal and multipotency. Downregulation of Sox2 decreases the expression of Sox1, neuroD, and NGN2, which are important genes in differentiation to neural cells. Interestingly, Modrek et al. ${ }^{31}$ have revealed that overexpression of the P53 mutation can rescue these dysfunctions. In addition, activation of PI3K/AKT signaling is associated with aggressive human glioma ${ }^{37}$. PI3K/AKT signaling regulates downstream genes such as podoplanin (PDPN) and retinol-binding protein 1 (RBP1). PDPN plays a considerable role in glioma pathogenesis ${ }^{34}$. Downregulation of PDPN has been observed in glioma carrying IDH1 mutations, which can increase chances for survival in patients carrying IDH1 ${ }^{\text {Mut } 38}$. Moreover, an IDH1 $1^{\text {Mut }}$ enhances AKT/mTOR activity. Since AKT/mTOR signaling is associated with cell migration, it may explain the high migration of IDH1 $1^{\text {Mut }}$ glioma cells ${ }^{36}$.

\section{Clinical prognostic value of IDH1 ${ }^{\text {Mut }}$}

The IDH1 mutation, O6-methylguanine-DNA methyltransferase (MGMT) promoter methylation, and 1p 19q deletion have been introduced ${ }^{39}$ as markers for a lowgrade glioma, anaplastic oligoastrocytoma, and glioblastoma, respectively, to predict sensitivity to chemotherapy and develop the appropriate prognosis. Patients who are positive for these three markers have a better progression-free survival ${ }^{36}$. After studying 1,010 patients, Hartmann et al. ${ }^{40}$ concluded that IDH1 mutations of the R132C type are strongly associated with astrocytoma, while IDH2 mutations mostly occur in oligodendroglial tumors. In addition, the IDH1 mutation occurs in younger patients ${ }^{39}$ which can be considered a predictor for grades 2 and 3, but not grade 4, glioma with a favorable prognosis, especially with radiation or alkylating therapy ${ }^{39,40}$. In this regard, the clinical trial demonstrated that patients with IDH1 mutations in grade 3 have a better response to chemotherapy, although chemo-radiotherapy may have a more positive effect on grades 2 and 3 tumors $^{41}$.

\section{IDH1 Mut and immunology in neuro-oncology}

IDH1-R132H is expressed in almost all slow-growing tumors, so the preparation of a vaccine targeting mutant IDH1 could be a novel therapeutic assay. Schumacher et al. found that IDH1-R132H contains an immunogenic epitope that can be used for vaccinations. They have constructed the artificial IDH1 polypeptides containing 15 amino acids. Injections of this construct with human major histocompatibility complexes (MHC) molecules in mice revealed that the mutated area could be presented on MHC class II that activated $\mathrm{CD}^{+}{ }^{+} \mathrm{T}$ helper- 1 and antibody production. This antibody can prevent the growth of IDH $1^{\text {Mut }}$ tumor cells without inhibiting the normal functions of wt IDH $1^{42}$. However, Weenink et $\mathrm{al}^{43}$ reported that they failed to detect any antibody specific to IDH1-R132H or CD4 ${ }^{+} \mathrm{T}$ cell reactivity in sera of the LGG patients. In another study, Pellegatta et al. transplanted murine glioma cells containing the IDH1 mutation in the brains of mice and then immunized them to different peptides encompassing the IDH1 mutation. The immunized mice demonstrated an increasing number of $\mathrm{CD}^{+} \mathrm{T}$ cells and IFN $\gamma$ production with an upregulation in the granzyme-b and perforin- 1 and a corresponding downregulation of TGF-B2 and IL10. They also detected an antibody against IDH1 ${ }^{\text {Mut }}{ }^{44}$. It has been shown that increasing the number of $\mathrm{T}$ cells may be due to a reduction of the expression of programmed deathligand 1 (PD-L1) in mutant IDH ${ }^{45}$. However, Kohanbash 
Table 1 Clinical trial of drugs or small molecules on gliomas containing the IDH1 mutation.

\begin{tabular}{|c|c|c|c|c|c|c|}
\hline Clinical trail.gov & $\begin{array}{l}\text { Phase } \\
\text { of trial }\end{array}$ & $\begin{array}{l}\text { Compound/ } \\
\text { treatment tool }\end{array}$ & Participants & Dosing & Result & Refs. \\
\hline NCT02454634 & I & $\begin{array}{l}\text { IDH1 } 20 \text { mer peptide } \\
\text { vaccine (NOA-16) }\end{array}$ & 39 & 8 times every $2 / 4$ weeks & $\begin{array}{l}\text { Cytotoxic immune response in and } \\
\text { humoral immune responses }\end{array}$ & 79 \\
\hline NCT02073994 & I & AG-120 & 170 & 28-day cycles, oral & Ongoing trail & 80 \\
\hline NCT02968940 & $\|$ & Avelumab + Radiation & 43 & Every 2 weeks & - & 81 \\
\hline NCT03212274 & $\|$ & Olaparib & 145 & 28-day cycles, oral & - & 81 \\
\hline NCT02746081 & 1 & BAY1436032 & 81 & 21-day cycles, oral & - & 82 \\
\hline NCT02771301 & I & IDH-R132H-DC vaccine & 30 & - & - & 83 \\
\hline NCT02481154 & । & AG-881 (ivosidenib) & 95 & 28-day cycles, oral & $\begin{array}{l}\text { Favorable safty at doses } 100 \mathrm{mg} \\
\text { and above }\end{array}$ & 84 \\
\hline NCT03343197 & $\|$ & $A G-120+A G-881$ & 49 & 28-day cycles, oral & Ongoing trail & 85 \\
\hline NCT02273739 & $|/|||$ & AG-221 & 21 & 28-day cycles, oral & - & 86 \\
\hline NCT02381886 & । & IDH305 & 166 & - & - & 87 \\
\hline NCT02193347 & । & IDH1 peptide vaccine & 24 & - & - & 88 \\
\hline NCT03681028 & । & AG-881 & & Oral & $\begin{array}{l}\text { Tumor growth } 6.8 \% \text { compared to } \\
24.5 \% \text { placebo }\end{array}$ & 89 \\
\hline NCT02209428 & $\|$ & Temozolomide & 54 & Every 21 days, 6 cycles. & - & 90 \\
\hline NCT03557359 & $\|$ & Nivolumab & 37 & $\begin{array}{l}\text { Every } 2 \text { weeks for } 8 \text { cycles } \\
\text { for } 2 \text { years, infusion }\end{array}$ & Ongoing trail & 91 \\
\hline NCT03960502 & I & AG-881 & 5 & $\begin{array}{l}\text { Single dose, oral followed } \\
\text { with infusion }\end{array}$ & - & 92 \\
\hline NCT04164901 & III & AG-881 & 366 & Daily dosing, oral & Ongoing trail & 93 \\
\hline NCT03684811 & $|/| \mid$ & FT-2102 + Azacitidin & 200 & - & Ongoing trail & 94 \\
\hline NCT03666559 & $\|$ & Azacitidine & 63 & $\begin{array}{l}\text { Seven consecutive days } \\
\text { every } 4 \text { weeks, injection }\end{array}$ & - & 95 \\
\hline
\end{tabular}

et al. ${ }^{46}$ have found that reduction of PD-L1 may not result in a stronger $\mathrm{T}$ cell response, because $\mathrm{D} 2 \mathrm{HG}$ inhibits ATP-dependent $\mathrm{T}$ cell receptor signaling, which in turn leads to suppression of $\mathrm{T}$ cell anti-tumor immunity. A summary of the main aspects of IDH1/IDH $1^{\text {MUT }}$ in glioma cell biology are depictured in Fig. 1.

\section{Clinical trials targeting IDH1 in brain tumors}

There are many experiments to discover new therapeutic medications such as small molecules or drugs to target IDH1/2 in low-grade gliomas conducted and some have progressed to validation in humans. These clinical trials are summarized in Table 1.

\section{Modeling glioma carrying IDH1 mutations}

There are many studies about the role of IDH1 and $\mathrm{R} 132 \mathrm{H}$ mutation in glioma. However, a more complete understanding of the contribution of this mutation in tumorigenesis, the identification of interconnecting pathways, and the exploitation of $\mathrm{IDH}^{\mathrm{Mut}}$-associated molecular and cellular alterations for diagnostic and therapeutic strategies have been hindered largely due to the lack of an appropriate model. One of the best ways to investigate different aspects of the role of the IDH1$\mathrm{R} 132 \mathrm{H}$ mutation is, providing in vitro and in vivo models. Since in vivo animal models are complex, expensive and time consuming, as well as ethically controversial, the establishment of in vitro models to study human tumorigenesis could be helpful.

In the view of the authors, one promising strategy is the generation of reductionistic molecular conditions that provide a tool to focus on specific aspects of tumor biology in an isogenic-controlled background. This reductionist model might be simple to study the effect of a single or multiple mutation(s) or mechanistic function and epigenetic modifications of these gene mutations in 
cancer. Other kinds of experimental models are those that aim to recapitulate the human disease conditions with all complexities. The cell of origin, as well as the type of transformation strategy, influences the feasibility of performing straightforward and functional assays ${ }^{21}$. The benefits and limitations of both approaches need to be considered and put in the context of the individual research question. We will include our perspective in this regard in the following model presentations.

\section{Procedures of in vitro modeling Patient-derived disease models}

In vitro modeling is an important procedure to understand developmental and disease mechanisms, as well as the preclinical testing of drugs. Glioma models carrying the IDH1 ${ }^{\text {Mut }}$, in vitro, can be based on the establishment of primary patient cells or generating IDH1 mutated cell lines via gene engineering or editing. In the following section, we will summarize the main findings made with classical serum-based cancer line, neurosphere cell lines, or primary cell models.

Classical serum-cultured glioblastoma cell lines as a receiver matrix for transforming elements: Human glioma cell lines derived from patients have been used as a tool to produce glioma models. The two most well-known glioma cell lines are $\mathrm{U} 87^{47}$ and $\mathrm{U} 251^{48}$. In spite of the long time culture, the genetic aberrations from the original tumors retained in these cell lines. Sequencing of the genome of U87 revealed an enormous number of indels, copy number variations, and translocations, which are most likely due to the condition of the serum culture ${ }^{49}$. Therefore, regular cell line verification is required. Moreover, passaging these cells in the medium containing serum differentiates U87 more to astrocyte ${ }^{21}$. However, due to easy gene manipulation and rapid culturing and maintenance of the original genetic aberrations, this glioma cell line has been used in several drugs in vitro screenings ${ }^{50}$. U87 and U251 have also been used to overexpress IDH1 wt and R132H, as well as to study the mechanism in glioma and the behavior of the cells. Zhu et al. transfected U87 to overexpress IDH1 wt, IDH1R132H (pCMV-TAG2B) by lipofectamine 2000. Overexpressed IDH1-R132H cells were more sensitive to 5-FU, meaning that this medication could increase apoptosis in mutated IDH1 due to the decreasing expression of $\mathrm{NADPH}$ and $\mathrm{CGH}$ as antioxidants ${ }^{36}$. Cui et al. also overexpressed IDH1 Wt and R132H (ligated in pLenti6.3MCS-IRES2-EGFP) by lentivirus in the U87 and U251 cell lines and showed the correlation between IDH1 mutations and B-catenin/Wnt pathways. Wnt/ $\beta$-Catenin pathways are involved in the metastasis of different cancers through the initiation of epithelial to mesenchymal transition $^{51}$, including glioblastoma. Overexpression of Wnt/B-Catenin in U87-IDH1-R132H and tumor samples carrying the $\mathrm{R} 132 \mathrm{H}$ mutation restored a decline of proliferation and a high apoptosis ratio, reducing both invasion and migration in vitro and in vivo in the mutated cells. They concluded that mutant IDH1 can play an antitumor role in glioma. $\mathrm{Li}$ et al. used the pLPCX vector to overexpress IDH1-wt, IDH1-R132H, and IDH2-R172K by retrovirus in U87 and U373. IDH1-R132H and IDH2R172K cells were more sensitive to radiation. This behavior was associated with a high rate of apoptosis and oxidative stress.

Primary cell models from patients suffering from tumors of IDH ${ }^{\text {Mut }}$ : Primary, low-grade glioma (LGG)derived cells survive in a neurobasal medium containing EGF and $\mathrm{bFGF}^{52}$. Piaskowski et al. tried to expand primary glioma cells carrying IDH1 mutations. They reported that glioma cells with IDH1 mutations cannot be cultured in vitro because of their death in standard cell culture condition ${ }^{53}$. Understanding the mechanism of this phenomenon can help the scientific community find new therapeutic targets.

The neurosphere assay is the gold standard for keeping stem cells in brain tissue ${ }^{54}$ and can help achieve better cultivation of mutant IDH glioma cells. Nevertheless, the behavior of the neurosphere depends on the genetics and grade of the tumor. Sphere formation can be the result of the anchorage-independent growth that happens due to oxidative stress ${ }^{55}$. Stoczynska-Fidelus et al. ${ }^{53}$ revealed that $3 \mathrm{D}$ cell culture increases the survival and proliferation rate of glioma cells. In contrast, Tiburcio et al. ${ }^{55}$ have demonstrated that neurosphere formation in heterogenous IDH1$\mathrm{R} 132 \mathrm{H}$ reduces the concentration of D2HG, compared with adherent culture. Recently, Jacob et al. ${ }^{56}$ established a patient-derived glioblastoma organoid (GBO) platform that can recapitulate parental tumor in vitro according to histological features, cellular diversity, gene expression, and mutational profiles. They dissected fresh tumor specimens into small pieces and established GBOs using neurosphere conditions in a shaking culture environment. They could generate organoids for $96.4 \%$ IDH1-wt and $66.7 \%$ with IDH1 mutations for over 48 weeks with a similar gene expression of parental tumor markers. Although they were successful in generating and testing glioma cells derived from patients, the efficiency for mutated IDH1 was low in comparison to LGG with IDH1 $\mathrm{wt}^{56}$. They concluded that more optimization is required to establish IDH1 mutated organoids. On the other hand, patient glioma cells can be reprogrammed to induce pluripotent stem cells (iPSC) by the overexpression of Yamanaka factors Oct4, Sox2, Cmyc and KLF4 $4^{57}$, or Oct4, Nanog, L28, and Sox $2^{58}$. Recently, Liu et al. overexpressed Yamanaka's transcription factors in LGG cells BTO1 (carrying the R132C mutation), BTO3 (carrying the R132H mutation), and BT-142 (ATCC \#ACS1018) that originated from a grade III oligoastrocytoma carrying the mono-allelic R132H mutation. Their results 
demonstrate that cells containing the IDH1 mutation are resistant to reprogramming. Their explanation is the high sensitivity of the pluripotent stem cells to $\mathrm{D} 2 \mathrm{HG}^{59}$ because when human embryonic stem cells (hESCs) were treated with D2HG for $24 \mathrm{~h}$, the hESCs could not tolerate D2HG and die. They found, however, that reprogrammed colonies showed genetic changes, such as amplification of chromosome Xq23 or chromosome 11 and deletion of chromosome 1p, 4, 19q, and $\mathrm{Y}$, amplification of $1.4 \mathrm{Mb}$ region on chromosome $7 \mathrm{q} 31$, with co-amplification of genes located in this region. Analysis of tumor cells derived from LGG samples in a serum-free medium showed that they carry Xq23, 7q31 amplification, 1p 19q deletion, and the IDH $1^{\text {Mut }}$, from which it can be concluded that these amplifications or deletions in primary patient cells carrying the IDH $1^{\text {Mut }}$ are perhaps early mutational events associated with the manifestation of the IDH1 ${ }^{\text {Mut59 }}$.

Cell lines expressing mutated IDH1 can be generated by gene targeting methods such as zinc finger (ZFN), TALEN (Transcription activator-like effector nuclease), or clustered, regularly interspaced short palindromic repeats (CRISPR) or by overexpressing of wild-type and IDH1$\mathrm{R} 132 \mathrm{H}$ in various cell lines. The background of different experimental strategies, such as cellular background, mutation strategy, model validation, and core principal observations to model gliomas carrying the IDH1 mutation is summarized in Tables 2-4, respectively.

\section{Healthy donor-derived disease models}

Although the origins of glioma are not well understood, gliomas are generally thought to arise from the neural stem cells or the populations of progenitor cells ${ }^{2}$. Neural stem cells (NSCs) present a stable genome when they propagate as neurospheres in serum-free medium ${ }^{60}$. Rosiak et al. used neural progenitor cells derived from human-induced pluripotent stem cells (hiPSC-NPCs) to express IDH1-R132H as a glioma model. They induced hiPSC-NPCs by a lentiviral construct containing the $\mathrm{R} 132 \mathrm{H}$ mutation under the EF1 promoter. Overexpression of IDH1-R132H in hiPSC-NPCs decreased the differentiation ability of these cells to neuronal and inhibited differentiation to glial cells ${ }^{61}$. In addition, they demonstrated that overexpression of IDH1-R132H enhanced apoptosis in induced hiPSC-NPCs and during differentiation $^{61}$. Apoptosis of the human glioma cells containing IDH1-R132H can be considered as a tumor suppressor by downregulating Wnt $/ \beta$-catenin signaling ${ }^{51}$.

Turcan et al. demonstrated that induction of immortalized human astrocytes (by pLNCX2in packaged in lentivirus) to overexpress IDH1-wt and $\mathrm{R} 132 \mathrm{H}$ can reshape methylome in the induced cells but in two different manners. Expression of IDH1-wt caused hypomethylation, while expression of IDH1-R132H hypermethylated CpG island in proneural, similar to what was observed in low- grade glioma (LGG) containing the IDH1-R132H mutation. They showed histone modification, such as hypermethylation of H3K9me2, H3K27me3 involved in hypermethylation of DNA, and declination of TET2dependent 5-hydroxymethylcytosine $(5 \mathrm{hmC})$ levels. In this study, a mechanistic basis for the accumulation of DNA-methylation that leads to hypermethylation of CpG island was explained ${ }^{62}$. Tiburcio et al. also showed that overexpression of heterozygous IDH1-R132H by lentivirus (pLenti6.3/TO/V5-EST) in IMA can reduce the glutathione-oxidized glutathione (GSH/GSSG) ratio in comparison to IDH1 hemizygous and wt neurospheres. Interestingly, this ratio was even lower in adherent IDH1$\mathrm{R} 132 \mathrm{H}$ cells in comparison to neurospheres. In this study, Tiburcio et al. ${ }^{55}$ concluded that reducing power can control neurosphere genesis and the Wt allele contributes to gaining reduction power. This result not only emphasized the effect of heterozygosity of IDH1 in D2HG production but also confirmed that the IDH1 mutation in glioma can have an anti-oncogenic role ${ }^{63}$, which is an early phenomenon in glioma development.

New recombinant genomic techniques, TALEN and CRISPR, have been used to generate IDH1 mutant cell lines or study the behavior of IDH1 mut. Wei et al. employed a modified CRISPR/Cas9 to generate heterozygous IDH1-R132H mutation in human astroglial cells by applying single base editing. They revealed methylome changes associated with downregulation or upregulation of particular genes. In this regard, many metabolic enzymes were downregulated via hypermethylation of the promoter, including acyl-CoA dehydrogenase (ACADS), Aldehyde Dehydrogenase 2 Family (ALDH2), and Aldehyde Oxidase 1 (AOX1), indicating the involvement of IDH1 in multiple energy pathways. In addition, they showed that hypermethylation of the promoter can lead to histone modification and declination of histone markers such as H3K27me3, H3K36me3, and H3K4me3 in IDH1-R132H cells ${ }^{64}$. Wei et al. also demonstrated increasing migration of cells by upregulation of integrin $\beta 4$ (ITGB4) and inhibition of the proliferation of mutant cells. Furthermore, Wei et al. introduced Yes-associated protein (YAP) and Notch signaling, two prominent phylogenetically conserved stem cell pathways, as a molecular target responsible for cell growth inhibition in IDH1$\mathrm{R} 132 \mathrm{H}$ cells. In this study, Wei et al. ${ }^{64}$ also showed that enhancement of the migration and invasion of IDH1$\mathrm{R} 132 \mathrm{H}$ cells promote dependence on D2HG.

Nowadays, hiPSC is used as a promising tool in disease modeling ${ }^{65}$. Human iPSC can generate the proper model for glioma modeling because of self-renewing and differentiation to different types of cells ${ }^{66}$. Nevertheless, the genome instability and epigenetic memory during the reprogramming process and iPSC maintenance remain unexplained. Due to the toxicity of 


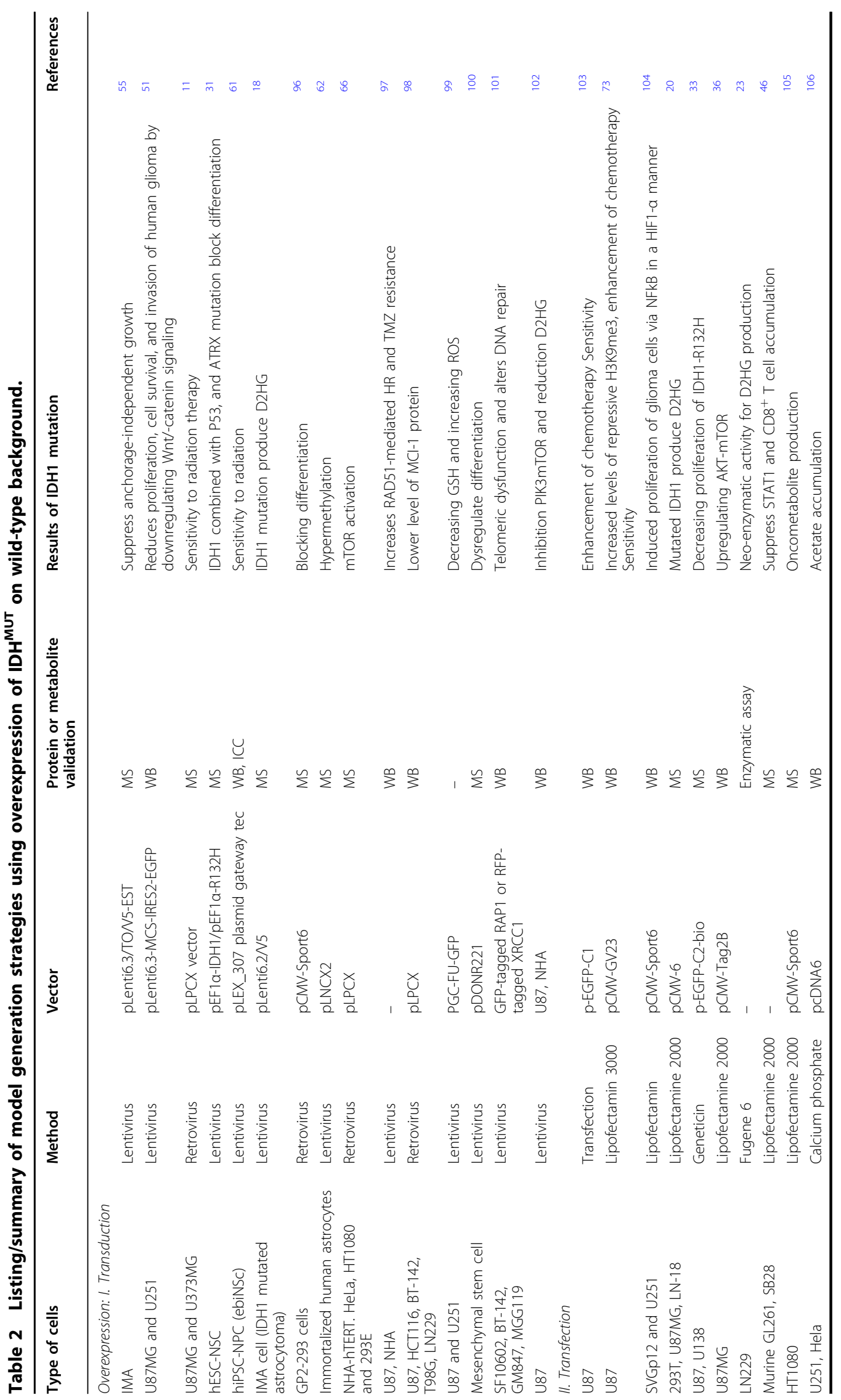


Table 3 Listing/summary of model generation strategies using gene targeting.

\begin{tabular}{|c|c|c|c|c|c|}
\hline HeLa cells & CRISPR & $\begin{array}{l}\text { gWIZ. } \\
\text { Luciferase }\end{array}$ & MS & $\begin{array}{l}\text { Deficient in } \\
\text { DNA repair }\end{array}$ & 107 \\
\hline Human iPSC & CRISPR & & MS & - & 70 \\
\hline SVG-10B1 & CRISPR & & MS & - & 64 \\
\hline HCT116 cell line & $H R$ & & MS & $\begin{array}{l}\text { IDH1-R132H alters } \\
\text { DNA-methylation }\end{array}$ & 108 \\
\hline
\end{tabular}

Table 4 Listing of model generation strategies applying successful reprogramming of patient cells.

\begin{tabular}{llll}
\hline Patient & Lentivirus & Yamanaka & - \\
glioma cells & factors & IDH1 mutation are not \\
& & reprogrammable \\
\hline
\end{tabular}

D2HG on hiPSCs ${ }^{59}$, scientists should investigate ways to by-pass this issue.

2D disease modeling by human iPSC can show some disease phenotypes, but there are no tissue or organ level structures. It is supposed that 3D modeling can demonstrate accurate pathophysiology of disease $\mathrm{e}^{67}$ and can recapitulate the in vivo environment ${ }^{52}$ because, in this condition, cells can express stemness genes. Laks et al. have shown that the spheroid culture of glioma primary cells expresses new genes related to malignancy and may help to culture primary cells carrying the IDH1 mutation. It is possible, however, that all spheres from primary glioma cells do not show a correlation with the parental tumor $^{68}$. Cerebral organoids derived from hiPSCs are a promising way to recapitulate diseases in vitro, such as GBM. Bian et al. ${ }^{69}$ established organoids expressing MYC-amplification (MYCOE), and organoids carrying CDKN2A-/CDKN2B-/EGFROE/EGFRvIIIOE, NF1-/ PTEN-/TP53- (p53), and EGFRvIIIOE/ CDKN2A-/ PTEN- called neoplastic cerebral organoids (neoCOR). Engraftment of neoCORs upon the renal subcapsular showed retaining and expansion of the organoid in the renal capsule with poor differentiation, as well as recapitulate tumorigenesis in vivo. NeoCORs could interact with normal organoids and tissue in the renal capsule, which would verify the invasiveness of the geneengineered organoids ${ }^{69}$.

Recently, Köpp et al. ${ }^{70}$ reported the gene modification of IDH1-R132H in hiPSC by CRISPR/Cas9 (efficiency 1\%). In light of the challenges associated with generating a CRISPmediated, iPSC-based IDH1 model, it will be interesting to see what functional validations of this model will be provided in the future. Initial results show that cerebral organoids from gene-modified iPSC-R132H are not affected in cell differentiation in the maturation of cerebral organoids. There are, however, some differences in cell growth between organoid DH1-Wt and IDH1-R132H.

In addition, recent work proved that iPSC-based creation of synthetic models for glioblastoma is a relevant strategy to study pathophysiological relevant tumor progression and to create molecular subtype-specific identities. We argue that using such an experimental design is a promising strategy that should be exploited in the search for IDH1-specific chemotherapies.

\section{In vivo models}

Most of our knowledge about the effect of IDH mutations in glioma were obtained from clinical studies or overexpression of the IDH1/2 mutation in various types of cells. Sasaki et al. generated the brain knock-in IDH1$\mathrm{R} 132 \mathrm{H}$ mouse model by the Cre-Laxp system. They induced mutated IDH1 in nestin-expressing cells and showed that this led to the death of mice directly after birth. Brain hemorrhage associated with accumulation of D2HG, high levels of hypoxia-inducible transcription factor-1a (HIF1 $\alpha$ ), reduction of ROS level, impaired collagen maturation, and disruption of basement formation were observed in mutant mice. They attributed these defects to the production of $\mathrm{D} 2 \mathrm{HG}^{71}$. In another study, Bardella et al. generated a mouse model of glioma by condition expressing IDH1-R132H in the subventricular zone (SVZ) in the brains of adult mice. These mice showed hydrocephalus and expanded lateral ventricles with an accumulation of D2HG and a reduction of $\alpha-K G$. NSCs isolated from these mice demonstrated a higher proliferation rate in vitro, and the SVZ area of the brain showed a high proliferation rate of these cells in vivo ${ }^{72}$. Recently, Nunez et al. generated a mouse model expressing IDH1$\mathrm{R} 132 \mathrm{H}$ associated with the deletion of p53 and ATRX. They showed an increase in the survival time without any treatment, as has been observed in patients. In addition, this study demonstrated hypermethylation of histone 3, which can cause epigenetic reprogramming and upregulation of the ataxia-telangiectasia-mutated (ATM) signaling pathway that leads to DNA damage response (DDR) as observed in the human glioma cells from surgical biop$\operatorname{sies}^{32}$. Moreover, the IDH1 mutation in this model resulted in radiotherapy resistance that could be restored by pharmacological inhibition of ATM or checkpoint kinases 1 and 2 , essential kinases in the DDR. Translation of these findings to patients with IDH1-R132H glioma and p53 and ATRX loss could help the therapeutic efficacy of radiotherapy and patient survival ${ }^{73}$.

\section{Discussion: perspectives of the authors}

To generate an in vitro model, the selection of a culture environment and the medium is a pivotal factor to recapitulate glioma. Since IDH1 mutations relate to both 
astrocytoma and oligodendroglioma, selecting the correct medium is essential to keep the IDH1 mutated model similar to the patient's condition, because using an inaccurate medium can change the sensitivity of cells to different drugs. For example, using NSC media for oligodendroglioma patient cells caused an omission of oligo precursor cells (OPC) properties and obtained high sensitivity to temozolomide wheeze, OPC medium supplemented with platelet-derived growth factor (PDGF) sustained OPC proliferation and development in vitro.

Glioblastoma, like other cancers, is a polygenic disease. Understanding cell behavior, or glioma mechanism, or analyzing each gene's role in glioma can help find the mechanism of glioma and targeting the pathway for treatment. On the other hand, one can combine the effect of multiple mutations related to glioma and understand the behavior of cells after these combinations. These models can be used as disease models to understand or screen various drugs on them. Human iPSCs are selfrenewal cells with the potential to differentiate into multiple kinds of cells, and relative genome stability may be a promising tool to recapitulate the disease in vitro. Generating in vitro models by overexpression of the wildtype or mutated IDH1 can be a way to establish the glioma model containing IDH1-R132H, although this is not an accurate model for disease modeling. Overexpression of the gene by transduction or transfection is based on random integration, in which the copy numbers and the location of the integrated gene cannot be controlled. The latter may attenuate or interfere with the expression of integrated genes or activate the oncogenes.

Reprogramming of glioma cells derived from patients and established isogenic clones is an alternative way to generate disease models for glioma-with or without IDH1 mutations. In this way, several isogenic clones should be selected to confirm cell characterization and metabolite production. Since D2HG production from mutated IDH1/2 can be a factor in inhibiting the reprogramming process, designing small molecules that can attach to the active catalytic site of mutated IDH1/2 may inhibit the mutated enzyme from producing D2HG instead of $\alpha$-KG. In this regard, IDH1 inhibitors (AG-120 ML $309^{74}, \mathrm{AG}-120^{75}$, and AGI-5198 ${ }^{76}$ ), and IDH2 inhibitors (AG-221 77 , AGI- $6780^{78}$ ) have shown that they can restore the IDH1 mutation effects ${ }^{17}$. Reprogrammed or overexpressed iPSCs or their derivations can differentiate and can be cultured in 2D or 3D. 3D cultures can recapitulate the organ structures. Brain organoids derived from human iPSCs (mini brain) are three-dimensional (3D) self-organized neural structures that can recapitulate the structure and development of the brain. Since cerebral organoids contain neuronal cells, astrocytes, and oligodendrocytes, they can be used to mimic astroglioma or oligodendroglioma. The high tendency of the cells carrying mutated IDH1 to grow as a sphere makes organoids a suitable model for understanding the pathophysiology of this mutation in glioma and may help us develop patient-specific therapies.

The fusion of cerebral organoids derived from iPSC and spheroid of patient glioma cells is a novel strategy to generate a glioblastoma model. Brain organoids may be able to fuse with glioma neurospheres or single glioma cells carrying the IDH1 mutation. This model can reveal the heterogenicity in glioma as well. Moreover, the interrogation of single-cell mutagenesis technologies in pluripotent or cancer cells targeting different diseaserelevant genes, either in a multiplex approach in one cell or each selected gene in one cell, and subsequent coculturing the differentially modulated cells in one organoid structure, while technologically realistic, remains a strategy on which little has been published to model the intra-tumoral heterogeneity of cancer in vitro.

One of the issues of organoid modeling is a short access to the vascular system. The lack of vascularization makes it very difficult for inner cells to have access to nutrients and gas exchange, which causes necrotic cells in the center of the organoid. Co-cultures of fused organoids or genemodified organoids with differentiated endothelial cells may solve this problem in disease modeling. Furthermore, using hydrogels can increase the diffusion of the nutrients and oxygen through organoid or 3D structures.

Co-cultures of patients, healthy $\mathrm{T}$ cells, or microglial or innate immune cells with fused organoids or organoid carrying mutated IDH1 can recapitulate cell infiltration in

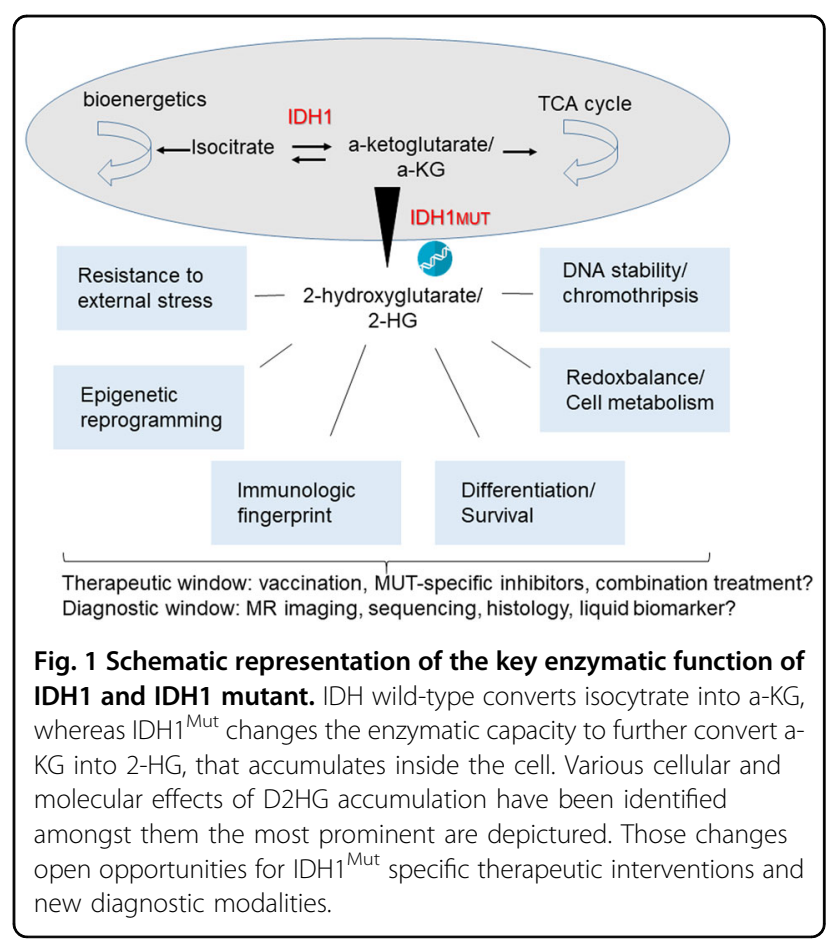


the brain. Another way to help generate the IDH1-R132H model could be through the transplantation of mutated IDH1 organoids in the brain of host animals as "in vivo bioreactors" to support the growth of cerebral organoids. Transplanted human brain organoids can differentiate neural and neuronal cells and integrate after transplantation in the cortex of mice. Infiltration of microglial, axon prolongation, and vascularization of the grafted organoids can also recapitulate the glioma. With growing evidence of the usefulness, and perhaps even the superiority of these modern cell culture technologies compared to animal models, we anticipate that the field will adopt those approaches as standard operating procedures in the near future. This is well in line with ethical considerations in science currently driving the $3 \mathrm{R}$ movement.

\section{Acknowledgements}

This work is financed by the German Federal Ministry of Education and Research (BMBFKZ 03VP0391) to U.D.K. Open Access funding enabled and organized by Projekt DEAL.

\section{Conflict of interest}

The authors declare that they have no conflict of interest.

\section{Publisher's note}

Springer Nature remains neutral with regard to jurisdictional claims in published maps and institutional affiliations.

Received: 3 August 2020 Revised: 29 October 2020 Accepted: 30 October 2020

Published online: 21 November 2020

\section{References}

1. Ludwig, K. \& Kornblum, H. I. Molecular markers in glioma. J. Neuro-Oncol. https://doi.org/10.1007/s11060-017-2379-y (2017).

2. Brat, D. J. et al. Comprehensive, integrative genomic analysis of diffuse lowergrade gliomas. N. Engl. J. Med. 372, 2481-2498 (2015).

3. Foote, M. B., Papadopoulos, N. \& Diaz, L. A. Genetic classification of gliomas: refining histopathology. Cancer Cell 28, 9-11 (2015).

4. Huang, J. et al. Isocitrate dehydrogenase mutations in glioma: From basic discovery to therapeutics development. Front. Oncol. 9, 1-7 (2019).

5. Kloosterhof, N. K., Bralten, L. B. C., Dubbink, H. J., French, P. J. \& van den Bent, M. J. Isocitrate dehydrogenase-1 mutations: a fundamentally new understanding of diffuse glioma? Lancet Oncol. 12, 83-91 (2011).

6. Krell, D. et al. Screen for IDH1, IDH2, IDH3, D2HGDH and I2HGDH mutations in glioblastoma. PLOS ONE 6, 3-6 (2011).

7. Sjöblom, T. et al. The consensus coding sequences of human breast and colorectal cancers. Science 314, 268-274 (2006).

8. Parsons, D. W. et al. An integrated genomic analysis of human glioblastoma multiforme. Science 321, 1807 (2008).

9. Singh, A., Gurav, M., Dhanavade, S., Shetty, O. \& Epari, S. Diffuse glioma-rare homozygous IDH point mutation, is it an oncogenetic mechanism? Neuropathology 37, 582-585 (2017).

10. $\mathrm{Mu}$, L. et al. IDH1 R132H mutation is accompanied with malignant progression of paired primary-recurrent astrocytic tumours. J. Cancer 8, 2704-2712 (2017).

11. Li, S. et al. Overexpression of isocitrate dehydrogenase mutant proteins renders glioma cells more sensitive to radiation. Neuro. Oncol. 15, 57-68 (2013).

12. Unruh, D. et al. Methylation and transcription patterns are distinct in IDH mutant gliomas compared to other IDH mutant cancers. Sci. Rep. 9, 1-11 (2019).

13. Horbinski, C. What do we know about IDH1/2 mutations so far, and how do we use it? Acta Neuropathol. 125, 621-636 (2013).
14. Amankulor, N. M. et al. Mutant idh1 regulates the tumor-associated immune system in gliomas. Genes Dev. 31, 774-786 (2017).

15. Al-Khallaf, H. Isocitrate dehydrogenases in physiology and cancer: biochemical and molecular insight. Cell Biosci. 7, 1-18 (2017).

16. Martínez-Reyes, I. \& Chandel, N. S. Mitochondrial TCA cycle metabolites control physiology and disease. Nat. Commun. 11, 1-11 (2020).

17. Kaminska, B., Czapski, B., Guzik, R., Król, S. K. \& Gielniewski, B. Consequences of $\mathrm{IDH} 1 / 2$ mutations in gliomas and an assessment of inhibitors targeting mutated IDH proteins. Molecules 24, 1-17 (2019).

18. Jin, G. et al. Disruption of wild-type IDH1 suppresses D-2-hydroxyglutarate production in IDH1-mutated gliomas. Cancer Res. 73, 496-501 (2013).

19. Garrett, M. et al. Metabolic characterization of isocitrate dehydrogenase (IDH) mutant and IDH wildtype gliomaspheres uncovers cell type-specific vulnerabilities. Cancer Metab. 6, 1-15 (2018).

20. Dang, L. et al. Cancer-associated $\mid \mathrm{DH} 1$ mutations produce 2hydroxyglutarate. Nature 462, 739-744 (2009).

21. Robertson, F. L., Marqués-Torrejón, M. A., Morrison, G. M. \& Pollard, S. M. Experimental models and tools to tackle glioblastoma. DMM Dis. Model. Mech. 12, dmm040386 (2019).

22. Yan, H. et al. Mutations in gliomas. N. Engl. J. Med. 360, 765-773 (2009).

23. Pusch, S. et al. D-2-hydroxyglutarate producing neo-enzymatic activity inversely correlates with frequency of the type of isocitrate dehydrogenase 1 mutations found in glioma. Acta Neuropathol. Commun. 2, 1-10 (2014).

24. Linninger, A. et al. Modeling the diffusion of D-2-hydroxyglutarate from IDH1 mutant gliomas in the central nervous system. Neuro. Oncol. 20, 1197-1206 (2018).

25. Ward, P. S. et al. The potential for isocitrate dehydrogenase mutations to produce 2-hydroxyglutarate depends on allele specificity and subcellular compartmentalization. J. Biol. Chem. 288, 3804-3815 (2013).

26. Kranendijk, M., Struys, E. A., Salomons, G. S., Van der Knaap, M. S. \& Jakobs, C. Progress in understanding 2-hydroxyglutaric acidurias. J Inherit Metab Dis 35, 571-587 (2012).

27. Yen, K. E., Bittinger, M. A., Su, S. M. \& Fantin, V. R. Cancer-associated IDH mutations: biomarker and therapeutic opportunities. Oncogene 29, 6409-6417 (2010).

28. Sun, Y. et al. A glioma classification scheme based on coexpression modules of EGFR and PDGFRA. Proc. Natl Acad. Sci. USA 111, 3538-3543 (2014).

29. Raineri, S. \& Mellor, J. IDH1: linking metabolism and epigenetics. Front. Genet. 9, 1-8 (2018).

30. Flavahan, W. A. et al. Insulator dysfunction and oncogene activation in IDH mutant gliomas. Nature 529, 110-114 (2016).

31. Modrek, A. S. et al. Low-grade astrocytoma mutations in IDH1, P53, and ATRX cooperate to block differentiation of human neural stem cells via repression of SOX2. Cell Rep. 21, 1267-1280 (2017).

32. Núñez, F. J. et al. IDH1-R132H acts as a tumor suppressor in glioma via epigenetic up-regulation of the DNA damage response. Sci. Transl. Med. 11, 1-14 (2019).

33. Bralten, L. B. C. et al. IDH1 R132H decreases proliferation of glioma cell lines in vitro and in vivo. Ann. Neurol. 69, 455-463 (2011).

34. King, D., Yeomanson, D. \& Bryant, H. E. PI3King the lock: targeting the PI3K Akt/mTOR pathway as a novel therapeutic strategy in neuroblastoma. J. Pediatr. Hematol/Oncol. 37, 245-251 (2015).

35. Tateishi, K. et al. PISK/AKT/mTOR pathway alterations promote malignant progression and xenograft formation in oligodendroglial tumors. Clin. Cancer Res. 25, 4375-4387 (2019).

36. $\mathrm{Zhu}, \mathrm{H}$. et al. IDH1 R132H mutation enhances cell migration by activating AKT-mTOR signaling pathway, but sensitizes cells to 5-FU treatment as NADPH and GSH are reduced. PLOS ONE 12, 1-13 (2017).

37. Peterziel, H. et al. Expression of podoplanin in human astrocytic brain tumors is controlled by the PI3K-AKT-AP-1 signaling pathway and promoter methylation. Neuro. Oncol. 14, 426-439 (2012).

38. Sun, C. et al. Wild-type IDH1 and mutant IDH1 opposingly regulate podoplanin expression in glioma. Transl. Oncol. 13, 100758 (2020).

39. Chen, $X$. et al. Clinical prognostic value of isocitrate dehydrogenase mutation, O-6-methylguanine-DNA methyltransferase promoter methylation, and 1p19q co-deletion in glioma patients. Ann. Transl. Med. 7, 541-541 (2019).

40. Hartmann, C. et al. Type and frequency of IDH1 and IDH2 mutations are related to astrocytic and oligodendroglial differentiation and age: a study of 1,010 diffuse gliomas. Acta Neuropathol. 118, 469-474 (2009).

41. Dang, L., Yen, K. \& Attar, E. C. IDH mutations in cancer and progress toward development of targeted therapeutics. Ann. Oncol. 27, 599-608 (2016). 
42. Schumacher, $T$. et al. A vaccine targeting mutant IDH1 induces antitumour immunity. Nature 512, 324-327 (2014).

43. Weenink, B. et al. Lack of B and T cell reactivity towards IDH1R132H in blood and tumor tissue from LGG patients. J. Neurooncol. 144, 79-87 (2019).

44. Pellegatta, S. et al. Effective immuno-targeting of the IDH1 mutation R132H in a murine model of intracranial glioma. Acta Neuropathol. Commun. 3, 4 (2015).

45. Han, S. et al. IDH mutation in glioma: molecular mechanisms and potential therapeutic targets. Br. J. Cancer 122, 1580-1589 (2020).

46. Kohanbash, G. et al. Isocitrate dehydrogenase mutations suppress STAT1 and CD8+ T cell accumulation in gliomas. J. Clin. Invest. 127, 1425-1437 (2017).

47. Pontén, J. \& Macintyre, E. H. Long term culture of normal and neoplastic human glia. Acta Pathol. Microbiol. Scand. 74, 465-486 (1968).

48. Westermark, B., Pontén, J. \& Hugosson, R. Determinants for the establishment of permanent tissue culture lines from human gliomas. Acta Pathol. Microbiol. Scand. Sect. A Pathol. 81A, 791-805 (2009).

49. Lenting, K., Verhaak, R., ter Laan, M., Wesseling, P. \& Leenders, W. Glioma: experimental models and reality. Acta Neuropathol. 133, 263-282 (2017).

50. Mikhailova, V. et al. Towards an advanced cell-based in vitro glioma model system. AlMS Genet. 5, 91-112 (2018).

51. Cui, D. et al. R132H mutation in IDH1 gene reduces proliferation, cell survival and invasion of human glioma by downregulating Wnt/B-catenin signaling. Int. J. Biochem. Cell Biol. 73, 72-81 (2016).

52. Ledur, P. F., Onzi, G. R., Zong, H. \& Lenz, G. Culture conditions defining glioblastoma cells behavior: what is the impact for novel discoveries? Oncotarget 8, 69185-69197 (2017).

53. Piaskowski, S. et al. Glioma cells showing IDH1 mutation cannot be propagated in standard cell culture conditions. Br. J. Cancer 104, 968-970 (2011).

54. Singec, l. et al. Defining the actual sensitivity and specificity of the neurosphere assay in stem cell biology. Nat. Methods 3, 801-806 (2006).

55. Tiburcio, P. D. B. et al. Functional requirement of a wild-type allele for mutant $\mathrm{IDH} 1$ to suppress anchorage-independent growth through redox homeostasis. Acta Neuropathol. 135, 285-298 (2018).

56. Jacob, F. et al. A patient-derived glioblastoma organoid model and biobank recapitulates inter- and intra-tumoral heterogeneity. Cell 180, 188-204.e22 (2020).

57. Takahashi, $\mathrm{K}$. et al. Induction of pluripotent stem cells from adult human fibroblasts by defined factors. Cell 131, 861-872 (2007).

58. $\mathrm{Yu}$, J. et al. Induced pluripotent stem cell lines derived from human somatic cells. Science 318, 1917-1920 (2007).

59. Liu, Z. et al. Characterization of iPSCs derived from low grade gliomas revealed early regional chromosomal amplifications during gliomagenesis. J. Neurooncol. 141, 289-301 (2019).

60. Reynolds, B. A. \& Weiss, S. Generation of neurons and astrocytes from isolated cells of the adult mammalian central nervous system. Science $\mathbf{2 5 5}$, 1707-1710 (1992).

61. Rosiak, K. et al. IDH1R132H in neural stem cells: differentiation impaired by increased apoptosis. PLOS ONE 11, 1-19 (2016).

62. Turcan, $\mathrm{S}$. et al. IDH1 mutation is sufficient to establish the glioma hypermethylator phenotype. Nature 483, 479-483 (2012).

63. Huang, L. E. et al. IGFBP2 expression predicts IDH-mutant glioma patient survival. Oncotarget 8, 191-202 (2017).

64. Wei, S. et al. Heterozygous IDH1 R132H/WT created by "single base editing" inhibits human astroglial cell growth by downregulating YAP. Oncogene 37, 5160-5174 (2018).

65. Bassett, A. R. Editing the genome of hiPSC with CRISPR/Cas9: disease models. Mamm. Genome 28, 348-364 (2017).

66. Carbonneau, M. et al. The oncometabolite 2-hydroxyglutarate activates the mTOR signalling pathway. Nat. Commun. 7, 12700 (2016).

67. Liu, C., Oikonomopoulos, A., Sayed, N. \& Wu, J. C. Modeling human diseases with induced pluripotent stem cells: From 2D to 3D and beyond. Dev 145, 1-6 (2018).

68. Laks, D. R. et al. Large-scale assessment of the gliomasphere model system. Neuro. Oncol. 18, 1367-1378 (2016).

69. Bian, S., Repic, M., Guo, Z., Kavirayani, A. \& Burkard, T. Europe PMC Funders Group Genetically engineered cerebral organoids model brain tumour formation. Nat Methods 15, 631-639 (2019).

70. Köpp, A et al. OS12.1 Editing of IDH1 R132H mutation in human induced pluripotent stem cells to investigate tumor genesis in glioma. NeuroOncology 21, iii22. https://academic.oup.com/neuro-oncology/articleabstract/21/Supplement_3/iii22/5564391?redirectedFrom=fulltext (2019).
71. Sasaki, M. et al. D-2-hydroxyglutarate produced by mutant ldh1 perturbs collagen maturation and basement membrane function. Genes Dev. 26, 2038-2049 (2012).

72. Bardella, C. et al. Expression of Idh1R132H in the murine subventricular zone stem cell niche recapitulates features of early gliomagenesis. Cancer Cell $\mathbf{3 0}$, 578-594 (2016).

73. Yin, N. et al. IDH1-R132H mutation radiosensitizes U87MG glioma cells via epigenetic downregulation of TIGAR. Oncol. Lett. 19, 1322-1330 (2020).

74. Davis, M. Probe Report: ML309, A Potent Inhibitor of R132H Mutant IDH1 Capable Of Reducing 2-Hydroxyglutarate Production in U87 MG Glioblastoma Cells 1-29 (National Center for Biotechnology Information, Bethesda, 2010).

75. Burris, H. et al. Abstract PL04-05: The first reported results of AG-120, a first-inclass, potent inhibitor of the IDH1 mutant protein, in a Phase I study of patients with advanced IDH1-mutant solid tumors, including gliomas. Mol. Cancer Therap. 14, PL04-05-PL04-05 (2015).

76. Rohle, D. et al. An inhibitor of mutant IDH1 delays growth and promotes differentiation of glioma cells. Science 340, 626-630 (2013).

77. Fan, B. Pharmacokinetic/pharmacodynamic evaluation of AG-120, a potent inhibitor of the IDH1 mutant protein, in a phase 1 study of IDH1-mutant advanced hematologic malignancies. EHA Library. 100713. https:/library.ehaweb.org/eha/2015/20th/100713/bin.fan.pharmacokinetic. pharmacodynamic.evaluation.of.ag-120.a.potent.html?f=m1 (2015).

78. Wang, F. et al. Targeted inhibition of mutant IDH2 in leukemia cells induces cellular differentiation. Science 340, 622-626 (2013).

79. NIH. Search of: NCT02454634 - List Results - ClinicalTrials.gov. https:// clinicaltrials.gov/ct2/results?cond $=\&$ term $=N C T 02454634 \&$ cntry $=\&$ state $=$ \&city $=\&$ dist $=$

80. NIH. Search of: NCT02073994 - List Results - ClinicalTrials.gov. https:// clinicaltrials.gov/ct2/results?cond $=\&$ term $=$ NCT02073994\&cntry $=\&$ state $=$ $\&$ city $=\&$ dist $=$

81. NIH. Search of: NCT03212274 - List Results - ClinicalTrials.gov. https:// clinicaltrials.gov/ct2/results?cond $=\&$ term $=$ NCT03212274\&cntry $=\&$ state $=$ $\&$ city $=\&$ dist $=$.

82. NIH. Search of: NCT02746081 - List Results - ClinicalTrials.gov. https:// clinicaltrials.gov/ct2/results?cond $=\&$ term $=\mathrm{NCT} 02746081 \&$ cntry $=\&$ state $=$ \&city $=\&$ dist $=$.

83. NIH. Search of: NCT02771301 - List Results - ClinicalTrials.gov. https:// clinicaltrials.gov/ct2/results?cond $=\&$ term $=$ NCT02771301\&cntry $=\&$ state $=$ \&city $=\&$ dist $=$.

84. NIH. Search of: NCT02481154 - List Results - ClinicalTrials.gov. https:// clinicaltrials.gov/ct2/results?cond $=\&$ term $=\mathrm{NCT0} 2481154 \&$ cntry $=\&$ state $=$ $\&$ city $=\&$ dist $=$.

85. NIH. Search of: NCT03343197 - List Results - ClinicalTrials.gov. https:// clinicaltrials.gov/ct2/results?cond $=\&$ term $=$ NCT03343197\&cntry $=\&$ state $=$ $\&$ city $=\&$ dist $=$.

86. NIH. Search of: NCT02273739 - List Results - ClinicalTrials.gov. https:// clinicaltrials.gov/ct2/results?cond $=\&$ term $=\mathrm{NCT} 02273739 \&$ cntry $=\&$ state $=$ $\&$ city $=\&$ dist $=$.

87. NIH. Search of: NCT02381886 - List Results - ClinicalTrials.gov. https:// clinicaltrials.gov/ct2/results?cond $=\&$ term $=$ NCT02381886\&cntry $=\&$ state $=$ $\&$ city $=\&$ dist $=$

88. NIH. Search of: NCT02193347 - List Results - ClinicalTrials.gov. https:// clinicaltrials.gov/ct2/results?cond $=\&$ term $=$ NCT02193347\&cntry $=\&$ state $=$ $\&$ city $=\&$ dist $=$.

89. NIH. Search of: NCT03681028 - List Results - ClinicalTrials.gov. https:// clinicaltrials.gov/ct2/results?cond $=\&$ term $=$ NCT03681028\&cntry $=\&$ state $=$ $\&$ city $=\&$ dist $=$.

90. NIH. Search of: NCT02209428 - List Results - ClinicalTrials.gov. https:// clinicaltrials.gov/ct2/results?cond $=\&$ term $=\mathrm{NCT} 02209428 \& \mathrm{cntry}=\&$ state $=$ $\&$ city $=\&$ dist $=$

91. NIH. Search of: NCT03557359 - List Results - ClinicalTrials.gov. https:// clinicaltrials.gov/ct2/results?cond $=\&$ term $=$ NCT03557359\&cntry $=\&$ state $=$ $\&$ city $=\&$ dist $=$.

92. NIH. Search of: NCT03960502 - List Results - ClinicalTrials.gov. https:// clinicaltrials.gov/ct2/results?cond $=\&$ term $=$ NCT03960502\&cntry $=\&$ state $=$ \&city $=\&$ dist $=$.

93. NIH. Search of: NCT04164901 - List Results - ClinicalTrials.gov. https:// clinicaltrials.gov/ct2/results?cond $=\&$ term $=$ NCT04164901\&cntry $=\&$ state $=$ $\&$ city $=\&$ dist $=$ 
94. NIH. Search of: NCT03684811 - List Results - ClinicalTrials.gov. https:// clinicaltrials.gov/ct2/results?cond $=\&$ term $=$ NCT03684811\&cntry $=\&$ state $=$ $\&$ city $=\&$ dist $=$.

95. NIH. Search of: NCT03666559 - List Results - ClinicalTrials.gov. https:/ clinicaltrials.gov/ct2/results?cond $=\&$ term $=$ NCT03666559\&cntry $=\&$ state $=$ \&city $=\&$ dist $=$.

96. Lu, C. et al. IDH mutation impairs histone demethylation and results in a block to cell differentiation. Nature 483, 474-478 (2012).

97. Ohba, S., Mukherjee, J., See, W. L. \& Pieper, R. O. Mutant IDH1-driven cellular transformation increases RAD51-mediated homologous recombination and temozolomide resistance. Cancer Res. $\mathbf{7 4}$ 4836-4844 (2014).

98. Karpel-Massler, G. et al. Induction of synthetic lethality in IDH1-mutated gliomas through inhibition of BCl-xL. Nat. Commun. 8, 1-13 (2017).

99. Shi, J. et al. Decreasing GSH and increasing ROS in chemosensitivity gliomas with IDH1 mutation. Tumor Biol. 36, 655-662 (2015).

100. Jin, Y. et al. Mutant idh1 dysregulates the differentiation of mesenchymal stem cells in association with gene-specific histone modifications to cartilage- and bone-related genes. PLOS ONE 10, 1-15 (2015).

101. Mukherjee, J. et al. Mutant IDH1 cooperates with ATRX loss to drive the alternative lengthening of telomere phenotype in glioma. Cancer Res. $\mathbf{7 8}$ 2966-2977 (2018).
102. Batsios, G. et al. PI3K/mTOR inhibition of IDH1 mutant glioma leads to reduced $2 \mathrm{HG}$ production that is associated with increased survival. Sci. Rep. $\mathbf{9}$ 1-15 (2019).

103. Wang, J. B., Dong, D. F., Wang, M. D. E. \& Gao, K. IDH1 overexpression induced chemotherapy resistance and $\mathrm{IDH} 1$ mutation enhanced chemotherapy sensitivity in glioma cells in vitro and in vivo. Asian Pac. J. Cancer Prev. 15, 427-432 (2014).

104. Wang, G. et al. Mutation of isocitrate dehydrogenase 1 induces glioma cell proliferation via nuclear factor-kB activation in a hypoxia-inducible factor 1-a dependent manner. Mol. Med. Rep. 9, 1799-1805 (2014).

105. Dexter, J. P. et al. Lack of evidence for substrate channeling or flux between wildtype and mutant isocitrate dehydrogenase to produce the oncometabolite 2-hydroxyglutarate. J. Biol. Chem. 293, 20051-20061 (2018).

106. Koyasu, S. et al. Increased 14C-acetate accumulation in IDH-mutated human glioblastoma: implications for detecting IDH-mutated glioblastoma with $11 \mathrm{C}$ acetate PET imaging. J. Neurooncol. 145, 441-447 (2019).

107. Sulkowski, P. L. et al. 2-Hydroxyglutarate produced by neomorphic IDH mutations suppresses homologous recombination and induces PARP inhibitor sensitivity. Sci. Transl. Med. 9, eaal2463 (2017).

108. Duncan, C. G. et al. A heterozygous IDH1R132H/WT mutation induces genome-wide alterations in DNA methylation. Genome Res. 22, 2339-2355 (2012) 